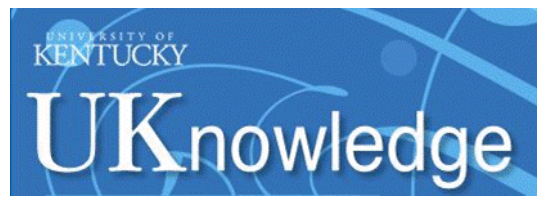

University of Kentucky

UKnowledge

\title{
A Hybrid Analytical and FE-Based Method for Calculating AC Eddy Current Winding Losses Taking 3D Effects into Account
}

\author{
Narges Taran \\ University of Kentucky, narges.taran@uky.edu \\ Dan M. Ionel \\ University of Kentucky, dan.ionel@uky.edu
}

Follow this and additional works at: https://uknowledge.uky.edu/peik_facpub

Part of the Power and Energy Commons

Right click to open a feedback form in a new tab to let us know how this document benefits you.

\section{Repository Citation}

Taran, Narges and Ionel, Dan M., "A Hybrid Analytical and FE-Based Method for Calculating AC Eddy Current Winding Losses Taking 3D Effects into Account" (2019). Power and Energy Institute of Kentucky Faculty Publications. 17.

https://uknowledge.uky.edu/peik_facpub/17

This Conference Proceeding is brought to you for free and open access by the Power and Energy Institute of Kentucky at UKnowledge. It has been accepted for inclusion in Power and Energy Institute of Kentucky Faculty Publications by an authorized administrator of UKnowledge. For more information, please contact UKnowledge@lsv.uky.edu. 


\title{
A Hybrid Analytical and FE-Based Method for Calculating AC Eddy Current Winding Losses Taking 3D Effects into Account
}

\author{
Digital Object Identifier (DOI) \\ https://doi.org/10.1109/ECCE.2019.8912784
}

\section{Notes/Citation Information}

Published in 2019 IEEE Energy Conversion Congress and Exposition (ECCE).

(C) 2019 IEEE Copyright Notice. "Personal use of this material is permitted. Permission from IEEE must be obtained for all other uses, in any current or future media,including reprinting/republishing this material for advertising or promotional purposes, creating new collective works, for resale or redistribution to servers or lists, or reuse of any copyrighted component of this work in other works."

The document available for download is the authors' manuscript version that is accepted for publication. The final published version is copyrighted by IEEE and will be available as: $N$. Taran, and D. M. Ionel, "A Hybrid Analytical and FE-based Method for Calculating AC Eddy Current Winding Losses Taking 3D Effects into Account," 2019 IEEE Energy Conversion Congress and Expo (ECCE), Baltimore, MD, 2019, pp. $1-6$. 


\section{A Hybrid Analytical and FE-based Method for Calculating AC Eddy Current Winding Losses Taking 3D Effects into Account}

\author{
Narges Taran, Student Member, IEEE \\ SPARK Lab, ECE Dept. \\ University of Kentucky \\ Lexington, KY, USA \\ narges.taran@uky.edu
}

\author{
Dan M. Ionel, Fellow, IEEE \\ SPARK Lab, ECE Dept. \\ University of Kentucky \\ Lexington, KY, USA \\ dan.ionel@ieee.org
}

\begin{abstract}
This paper proposes a new hybrid analytical and numerical FE-based method for calculating ac eddy current losses in wire windings and demonstrates its applicability for axial flux electric machines. The method takes into account 3D field effects in order to achieve accurate results and yet greatly reduce computational efforts. It is also shown that hybrid methods based on 2D FE models, which require semi-empirical correction factors, may over-estimate the eddy current losses. The new 3D FE-based method is advantageous as it employs minimum simplifications and considers the end turns in the eddy current path, the magnetic flux density variation along the effective length of coils, and the field fringing and leakage, which ultimately increases the accuracy of simulations. Case studies of axial flux PM motors: one with concentrated windings and open slots and another one with a coreless topology, are included.
\end{abstract}

Index Terms-Eddy current loss, ac winding loss, finite element, 3D model, PM machine, axial flux motor.

\section{INTRODUCTION}

The accurate prediction of the power loss components plays a vital role in the effective optimal design of electric machines, and, in this respect, ac eddy current winding losses are very important, especially in high-speed and high-power density designs. In this case, the nonlinearity caused by the large magnetic loading may increase the slot opening flux leakage and result into winding losses even at open circuit in addition to increased losses due to the proximity effects at load operation [1]-[3].

Analytical and numerical methods for estimating such ac winding losses have been previously developed and published, e.g. [4]-[7]. Analytical methods are more straightforward to use, but typically employ many simplifying assumptions, leading to approximate results. Numerical techniques, such as finite element analysis (FEA), may be more accurate with the downside of a laborious set-up process and substantial computer resource requirements.

In order to bridge the gap, hybrid methods have been proposed [2], [8], [9]. These methods generally employ the FEA calculated flux density in one coil cross section in order to provide a trade-off between accuracy and computational speed. On the other hand, due to end effects, flux leakage and fringing, the flux density observed by the winding in different cross sections may not be identical and hence employing a 2D FEA model may not suffice. For machines with considerable flux leakage at the ends, hybrid methods that utilize 2D FE models can overestimate the losses. Additionally, the accuracy is negatively affected because $2 \mathrm{D}$ models cannot take into account the end path of the eddy currents. The possible problems associated with sampling the flux density from a 2D FE model are discussed in more detail later in the paper. Furthermore, it should be noted that electrical machines, such as those of the axial flux type, in which the flux density in the slots and winding conductors vary both in the axial and radial directions, have a substantially three dimensional magnetic flux path and require adequate 3D FE models.

This paper proposes a new hybrid method with minimal simplifying assumptions. The analytical formulation is derived with the corresponding equations and the results are compared with meticulous 3D FEA of models with the windings detailed wire-by-wire. The method is illustrated on example axial flux permanent magnet (AFPM) machines with concentrated coils around the teeth and open slots, and with a coreless stator structure, respectively.

\section{NUMERICAL MODELS}

Two- and three-dimensional FEA models can be used to estimate the flux density and the losses. Two-dimensional FEA is easier to set up and is faster, making it particularly suitable for large-scale parametric and optimization studies. However, a major disadvantage is that it cannot take into account the variations in the third dimension. On the other hand, threedimensional models can take end effects and 3D flux paths into account, but have the drawbacks of being laborious to set up and computationally expensive.

It is possible to model the coils in detail, in a turn-by-turn and wire-by-wire approach in order to numerically calculate the ac winding losses. However, this is a complicated task, difficult to parametrize and to employ in an optimization algorithm. The winding ac loss estimation requires fine meshing 


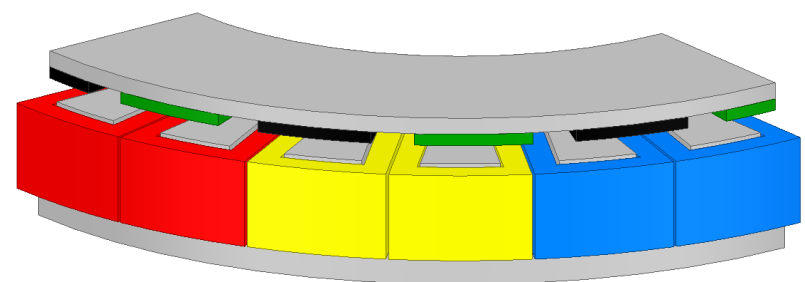

(a)

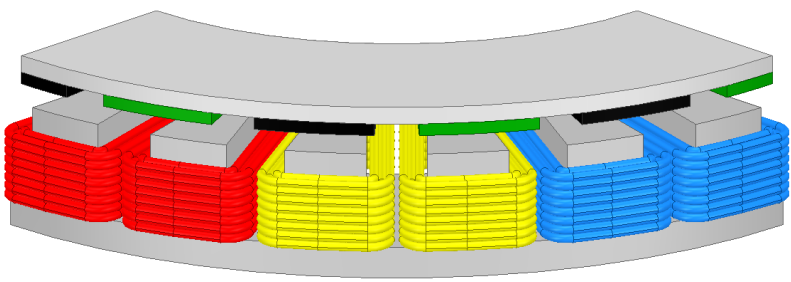

(b)

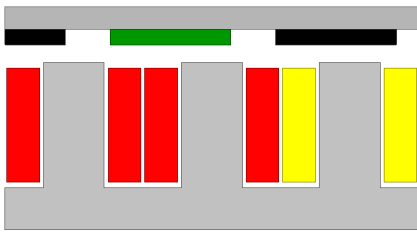

(c)

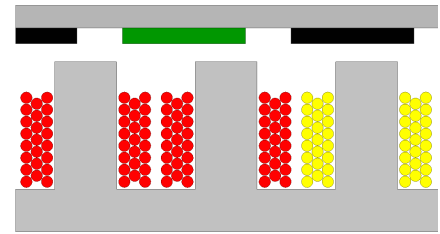

(d)
Fig. 1: The geometries employed for the FE models of the AFPM machine considered in the study. (a) Simplified 3D model with large equivalent single turn coil, (b) 3D model with turn-by-turn representation of wire conductors, (c) simplified 2D model with large equivalent single turn coil, and (d) 2D model with detailed turn-by-turn representation of the wire conductors.

according to the skin depth, while the flux density can be estimated with a more coarse mesh.

Four types of geometries employed throughout this paper for the FEA modeling of the example slotted AFPM machine studied are shown in Fig. 1. These include 2D and 3D models with a general large equivalent single turn coil, Figs 1a and $1 \mathrm{c}$, and detailed turn-by-turn models of the conductors, Figs. $1 \mathrm{~b}$ and $1 \mathrm{~d}$. The example 2D FEA results from Fig. 2 illustrate the flux lines and fringing as well as the induced eddy currents and losses in open-circuit.

\section{Hybrid Analytical-FEA Methods}

Hybrid methods may provide, in principle, a satisfactory compromise between accuracy and computational efforts for estimating the ac eddy current component of copper losses. For such methods, a combination of analytical equations and FEA is employed. Typically, flux density values are sampled from the simplified coil cross section representation of a $2 \mathrm{D}$ FE model, e.g. shown in Fig. 1c. The flux density is then used with analytical equations in order to calculate the eddy current losses.

Although hybrid methods may lead to more accurate estimations than pure analytical methods, they have the risk of inaccuracy due to the fact that they disregard 3D effects. Neglecting end paths, as shown in Fig. 3 may result into un-

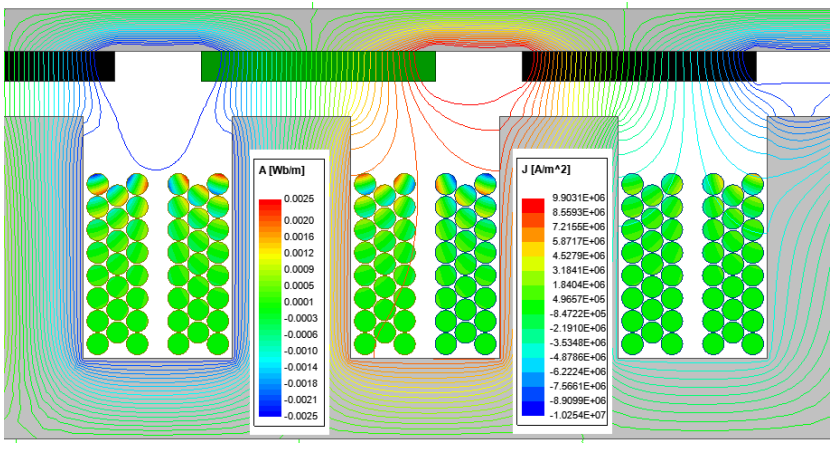

(a)

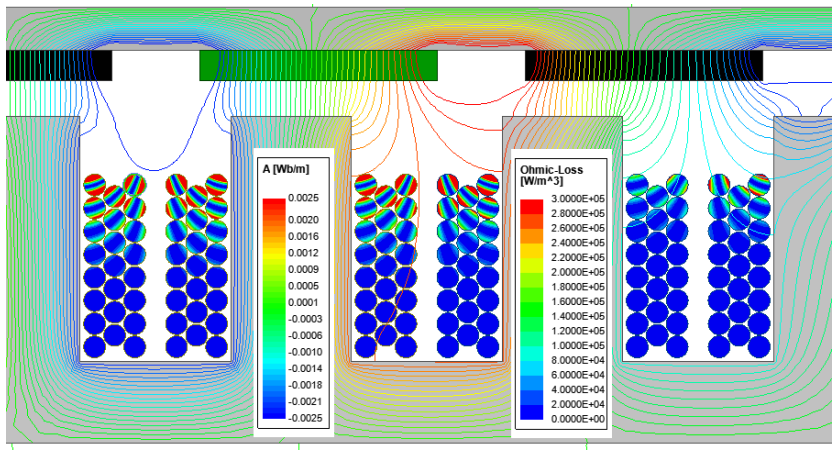

(b)

Fig. 2: The magnetic flux lines together with the induced eddy current density (a) and the power density losses in the conductors due to the eddy currents (b), respectively.

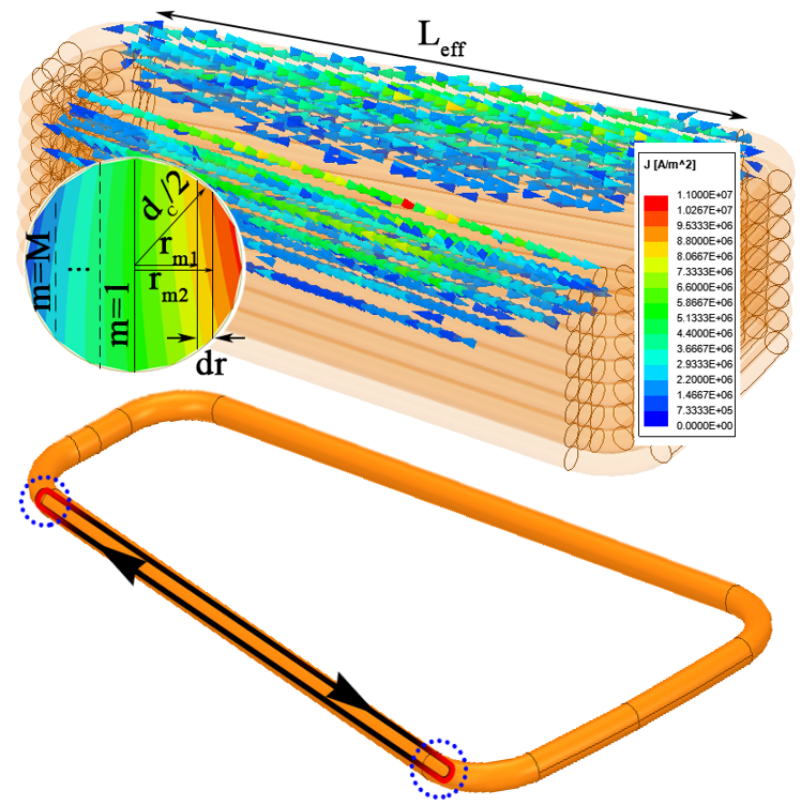

Fig. 3: Eddy current 3D distribution in the conductors (top) and a typical eddy current path considered in a $2 \mathrm{D}$ analysis, having a go and return path along the conductor $L_{e f f}$ and not including an end coil section.

derestimation of resistance and hence overestimation of eddy current losses. The end turn paths become a larger contributor to the resistance of the eddy current paths for shorter coils, i.e., 
lower stack length in case of radial flux machines or larger split ratio in case of axial flux machines. Therefore, it is necessary to consider the entire path of the eddy current loop. One typical approach is to employ correction factors, such as:

$$
K_{s}=1-\frac{\tanh \left(\frac{\pi L_{e f f}}{d_{c}}\right)}{\frac{\pi L_{e f f}}{d_{c}}}
$$

where $L_{e f f}$ is the effective length of the coil and $d_{c}$ is the conductor diameter. The above formulation of the correction factor, $K_{s}$, has been originally introduced for the calculation of eddy current losses in the screened-rotor of an induction motor [10] and later adopted for the rotor retaining can of PM excited machines [11]. In our study, $K_{s}$ is employed for calculating winding eddy current losses.

Another important reason for a possible overestimation of eddy current conductor losses by 2D methods may be attributed to not considering the magnetic field fringing and leakage, and hence neglecting the reduction of the flux density towards the ends, as illustrated in Fig. 4. Hybrid methods that employ 2D models ignore the fringing in the third dimension and typically result in larger and constant values of the flux density along an entire coil side. Moreover, for axial flux machines the non-linear magnetic field along the radial direction can largely vary and sampling the flux density at only one particular diameter may not be truly representative of the flux density throughout the entire coil.

For an example AFPM machine, the magnitude of the flux density in each of the winding turns placed in the slot and surrounding a tooth was estimated with one sample per conductor using 2D and 3D models, respectively (Fig. 6). The 3D samples are taken for each of the 21 turns placed at equally distanced radial locations and then averaged. The 2D model was set-up for the mean diameter. The 2D model overestimation of the flux density, particularly for the turns that are closer to the top of the slot and closer to the teeth, is noticeable.

The coreless AFPM machine example from Fig. 5 is illustrative of the typical very large $3 \mathrm{D}$ variation of the flux density in the stator windings. In order to account for this, one solution would be to study multiple 2D models representative of slices at different radial coordinates and combine their contributions [12]. An increased number of 2D slices would increase, in principle, the accuracy of the simulation at the expense of increased computational time, but won't still account for the end field, which makes the use of 3D models worthwhile even more so.

\section{Proposed Method}

The new method proposed in the paper employs a simplified 3D FE model with a single equivalent turn per coil as shown in Fig. 1a. The model can be solved as a magnetostatic field problem or as an eddy current problem, but with an ideal zero conductivity set for the conductor. The motivation for this approach is that the mesh required for satisfactorily accurate flux density calculations is substantially less dense

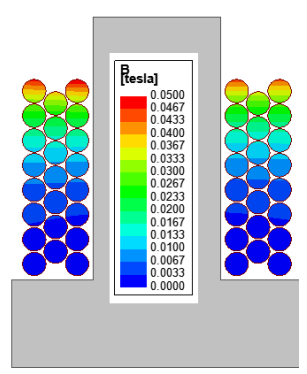

(a)

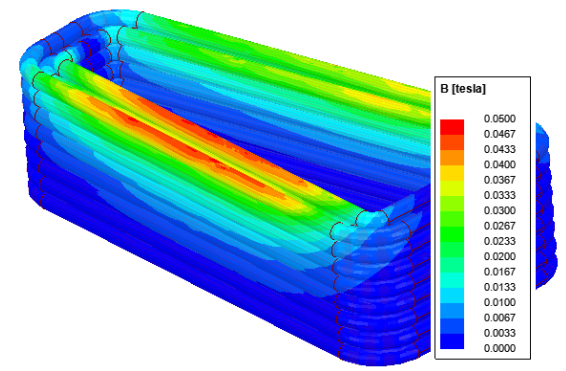

(b)
Fig. 4: The magnetic flux density in the conductors of a concentrated precise wound coil placed in the slots of an example AFPM machine calculated with 2D (a) and 3D (b) FEA and illustrating the multi-dimensional variation of the field.

than required for eddy current calculations in the winding conductors, resulting in faster solving and significantly lower computational resource requirements.

Considering $N_{L}$ sections in the radial direction and a precise winding configuration, as shown in Fig. 4:

$$
\begin{aligned}
& P_{n_{\phi}, n_{z}, n_{L}}=\left[x_{n}, y_{n}, z_{n}\right] ; \\
& n_{\phi}=1, \ldots N_{\phi} ; n_{z}=1, \ldots, N_{z} ; n_{L}=1, \ldots, N_{L} ; \\
& x_{n}=\frac{I D}{2} \cos \left(\frac{\theta_{i n}}{2}\right)\left(1-\frac{n_{L}}{N_{L}}\right)+\frac{O D}{2} \cos \left(\frac{\theta_{\text {on }}}{2}\right) \frac{n_{L}}{N_{L}} ; \\
& y_{n}=\frac{I D}{2} \sin \left(\frac{\theta_{\text {in }}}{2}\right)\left(1-\frac{n_{L}}{N_{L}}\right)+\frac{O D}{2} \sin \left(\frac{\theta_{\text {on }}}{2}\right) \frac{n_{L}}{N_{L}} ; \\
& \theta_{\text {in }}=\theta_{i}+2 \arcsin \left(\frac{d_{c}\left(2 n_{\phi}-1\right)}{I D}\right) ; \\
& \theta_{\text {on }}=\theta_{o}+2 \arcsin \left(\frac{d_{c}\left(2 n_{\phi}-1\right)}{O D}\right)
\end{aligned}
$$

where $P_{n_{\phi}, n_{z}, n_{L}}$ are the Cartesian coordinates of the $n^{\text {th }}$ point to be sampled for the flux density value; $N_{L}$, the number of sampling planes stacked in the direction of the effective length of the coil with an equal distance of $\Delta L=\frac{L_{e f f}}{N_{L}}$ between them; $N_{\phi}$ and $N_{z}$, the number of turns in the circumferential and axial directions, respectively, as shown in Fig. 7a; $I D$ and $O D$ the inner and outer diameters; $\theta_{i}$ and $\theta_{o}$ the inner and the outer coil span angles, as illustrated in Fig. 7b.

It should be noted that for random windings, methods such as the one described in [2] maybe further developed for 3D application in conjunction with the new techniques described in this paper. Also, it is important to carefully adapt the flux density sampling to the problem. For example, if the real conductor dimensions are larger than the skin depth and the variations of flux density inside each conductor may be considerable, multiple flux samples in each plane are needed.

The eddy current losses for a circular conductor can be calculated based on a general analytical formulation:

$$
P_{e d d y}=\frac{1}{R}\left(\frac{d \Phi}{d t}\right)^{2} ; d R=\frac{\left(L_{e f f}+2 r\right) \rho}{\sqrt{\frac{d_{c}^{2}}{4}-r^{2}} d r}
$$




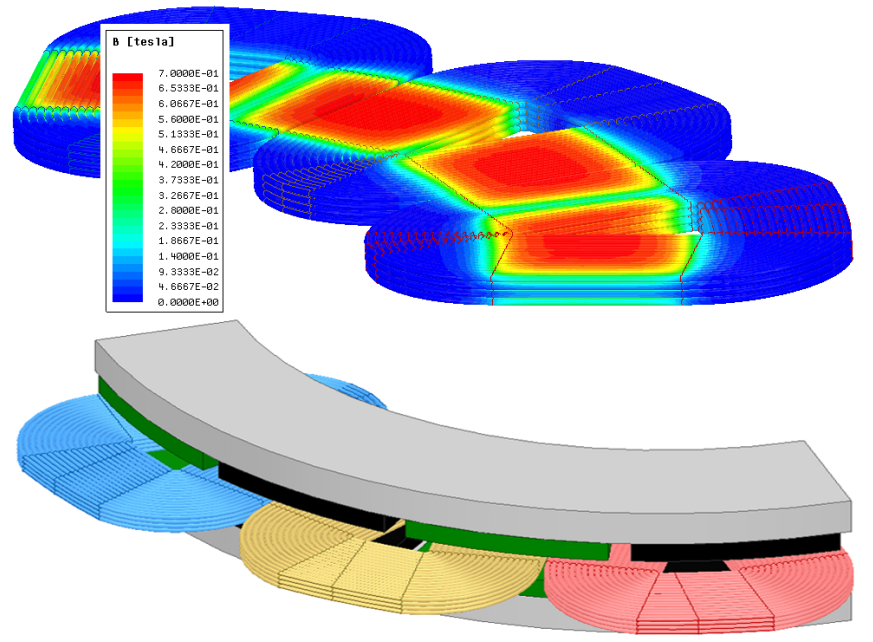

Fig. 5: The magnetic flux density in the conductors of an example AFPM coreless machine calculated with 3D FEA. For such machines, the winding is directly exposed to the airgap field, the magnitude and multi-dimensional variation of which can be substantial, resulting in significant eddy current losses.

where $R$ is the resistance; $\Phi$, the magnetic flux through the conductors; and $\rho$ the conductor resistivity. Each conductor cross section can be divided into $M$ segments as shown in Fig. 3. Assuming constant flux density for each segment, one field sample per segment may suffice.

Therefore, the eddy current loss for one coil side with $N_{\phi} \times$ $N_{z}$ turns can be estimated as:

$$
\begin{aligned}
& P_{e d d y}=\frac{4 L_{e f f}^{2}}{\rho} \sum_{n_{\phi}=1}^{N_{\phi}} \sum_{n_{z}=1}^{N_{z}} \sum_{h=1}^{\infty} \sum_{m=1}^{M} \ldots \\
& {\left[\left(\frac{d}{d t}\left(\frac{1}{L_{e f f}} \int_{I D}^{O D} B_{m, h}\left(\frac{D}{2}, n_{\phi}, n_{z}\right) d D\right)\right)^{2} \int_{r_{m 1}}^{r_{m} 2} \frac{r^{2} \sqrt{\frac{d_{c}^{2}}{4}-r^{2}}}{\left(L_{e f f}+2 r\right)} d r\right.} \\
& =\frac{L_{e f f}}{2 \rho} K_{s} \sum_{n_{\phi}=1}^{N_{\phi}} \sum_{n_{z}=1}^{N_{z}} \sum_{h=1}^{\infty} \sum_{m=1}^{M} \ldots \\
& {\left[\left(\frac{d}{d t}\left(\frac{1}{L_{e f f}} \int_{I D}^{O D} B_{m, h}\left(\frac{D}{2}, n_{\phi}, n_{z}\right) d D\right)\right)^{2}\left(f\left(r_{m 2}\right)-f\left(r_{m 1}\right)\right)\right]} \\
& f(r)=r \sqrt{\frac{d_{c}^{2}}{4}-r^{2}}\left(2 r^{2}-\frac{d_{c}^{2}}{4}\right)+\left(\frac{d_{c}^{2}}{4}\right)^{2} \arctan \frac{r}{\sqrt{\frac{d_{c}^{2}}{4}-r^{2}}}
\end{aligned}
$$

where $B_{m, h}$ is the $h^{t h}$ harmonic of the flux density in the $m^{t h}$ section for the conductor associated with $n_{\phi}$ and $n_{z}$ at the diameter $D$. The flux density of the $m^{t h}$ section includes the flux density of all sections that are inscribed in it (i.e. $\left.B_{m}=\sum_{m i=1}^{m} B_{m i}\right)$.

If the conductors are sufficiently small, the flux density throughout the conductor cross section may be assumed constant. Taking the variation of $B$ along the effective length from inner to outer diameter into account, and neglecting the harmonics content, the eddy current losses of the machine with $N_{c}$ coil sides are derived as:

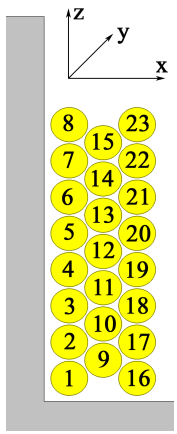

(a)

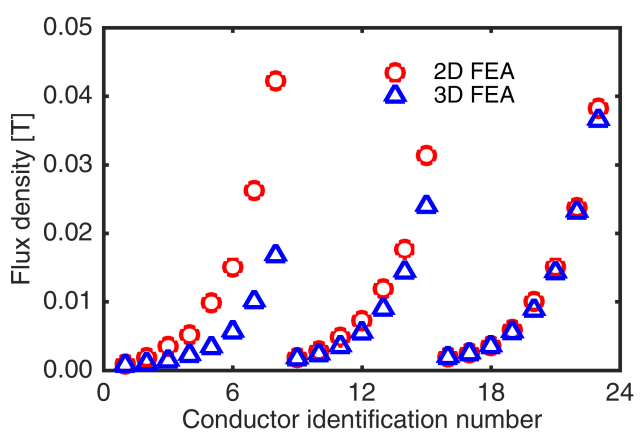

(b)
Fig. 6: (a) Schematic of a coil side placed around the tooth displaying conductor identification numbers. (b) The flux density in each conductor, obtained by space sampling 2D and 3D FEA results, respectively. The 2D FEA typically results in an over estimation, especially for the conductors at the top slot and closest to the tooth.

$$
\begin{aligned}
& P_{e d d y}=\frac{\pi L_{e f f} N_{c} d_{c}^{4} \omega_{e}^{2} K_{s}}{128 \rho} \ldots \\
& \sum_{n_{\phi}=1}^{N_{\phi}} \sum_{n_{z}=1}^{N_{z}}\left[\frac{1}{L_{e f f}} \int_{I D}^{O D} B\left(\frac{D}{2}, n_{\phi}, n_{z}\right) d D\right]^{2} .
\end{aligned}
$$

A discrete sampling along radial direction with $N_{L}$ samples can be performed in which case the coordinates of the sample points in the general 3D FEA model can be obtained from (2).

Both (4) and (5) assume that within the coil side the largest eddy current flow path follows the entire active length of the coil with shorter paths being possible, as shown in Fig. 8. The following equation, in which different eddy current paths inside one conductor are specified with the index $k$, takes this effect into consideration:

$$
\rfloor_{P_{e d d y}}=\frac{\pi L_{e f f} N_{c} d_{c}^{4} \omega_{e}^{2} K_{s}}{128 \rho} \sum_{n_{\phi}=1}^{N_{\phi}} \sum_{n_{z}=1}^{N_{z}} \sum_{k=1}^{k_{\max }} C_{k} \cdot B_{\text {path }, k}^{2},
$$

where $C_{k}$ is the coefficient adjusting the resistance of each current path and $B_{\text {path }, k}$ is the flux density within the $k^{t h}$ current path. In the case of an odd number of samples, $N_{L}$, along the coil side:

$$
\begin{aligned}
& k_{\text {max }}=\frac{N_{L}+1}{2}, \quad C_{k}=\frac{2+4(k-1)}{N_{L}}, \\
& B_{\text {path }, k}=\frac{1}{1+2(k-1)} \sum_{n=\frac{N_{L}+1}{2}-(k-1)}^{\frac{N_{L}+1}{2}+(k-1)} B_{n} .
\end{aligned}
$$

For an even number of samples:

$$
\begin{aligned}
& k_{\text {max }}=\frac{N_{L}}{2}, \quad C_{k}=\frac{4 k}{N_{L}}, \\
& B_{\text {path }, k}=\frac{1}{2 k} \sum_{n=\frac{N_{L}}{2}-(k-1)}^{\frac{N_{L}}{2}+k} B_{n} .
\end{aligned}
$$


TABLE I: ADDITIONAL AC COPPER LOSSES IN THE WINDINGS DUE TO EDDY CURRENTS, CALCULATED WITH DIFFERENT FEA AND HYBRID METHODS FOR OPEN-CIRCUIT AND FULL LOAD OPERATING CONDITIONS.

\begin{tabular}{llrrrr}
\hline AFPM machine & Eddy current loss & 3D FEA & Hy-3D & 2D FEA & Hy-2D \\
\hline Coreless & Open-circuit [W] & 420.5 & 424.0 & 611.7 & 520.3 \\
Open slot & Open-circuit [W] & 35.7 & 36.7 & 41.4 & 39.5 \\
Open slot & Full load [W] & 44.7 & 48.5 & 57.0 & 49.4 \\
Approximate FEA computational time & 37 hours & 35 minutes & 2 minutes & 12 seconds \\
\hline
\end{tabular}

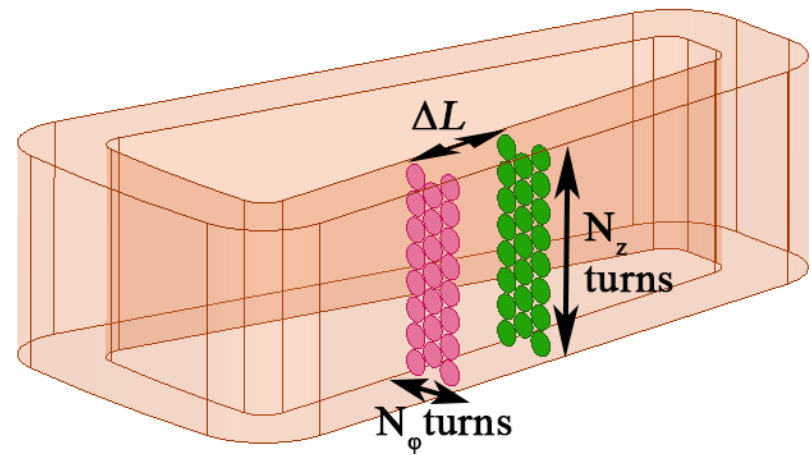

(a)

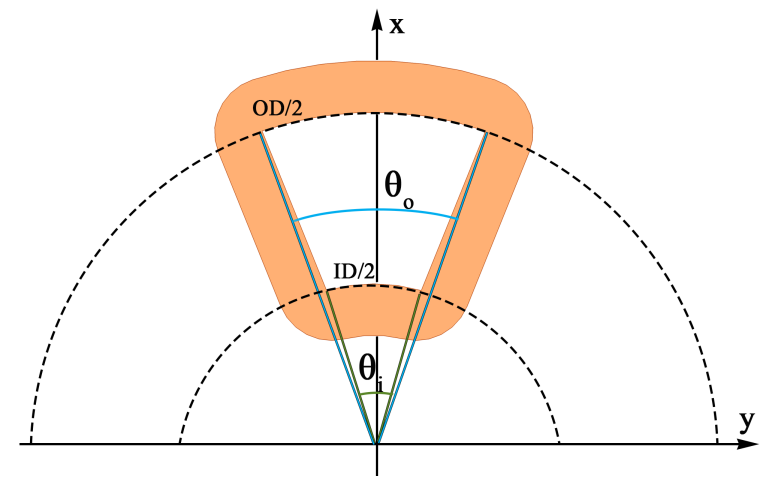

(b)

Fig. 7: (a) The flux density sampling planes employed by the new hybrid 3 FEA method stacked in the direction of the main current flow. (b) Threedimensional sampling is performed also in order to take end effects into account. The axial cross section schematic depicts the coil span and the in inner and outer diameter as used in (2).

Equation (6), which comprehensively represents the discrete implementation of the proposed new hybrid 3D FEA method, was employed in the following case studies.

\section{Case Studies}

Two example case studies were conducted: a 20 pole 24 slot AFPM machine with open slots and precise-wound concentrated windings with coils placed around the core teeth, as shown in Fig. 1, and a coreless AFPM machine with 12 coils and 16 poles, as shown in Fig. 5. The winding conductors were connected in series. It should be noted that in the case of parallel connections between turns, conductor transposition may reduce the circulating currents and additional losses.

The calculations were performed for open-circuit and load, at a fundamental frequency of $1.6 \mathrm{kHz}$ for the open-slot machine and at $480 \mathrm{~Hz}$ for the coreless design, respectively.

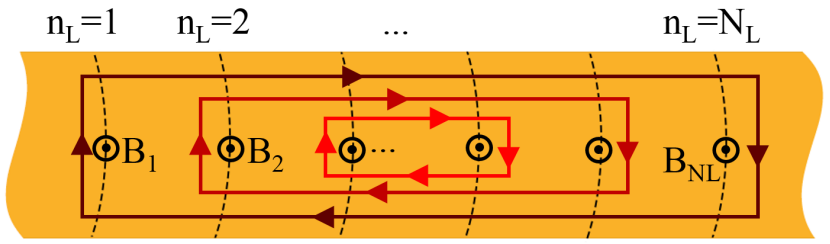

Fig. 8: Eddy current paths with different length along the coil side.

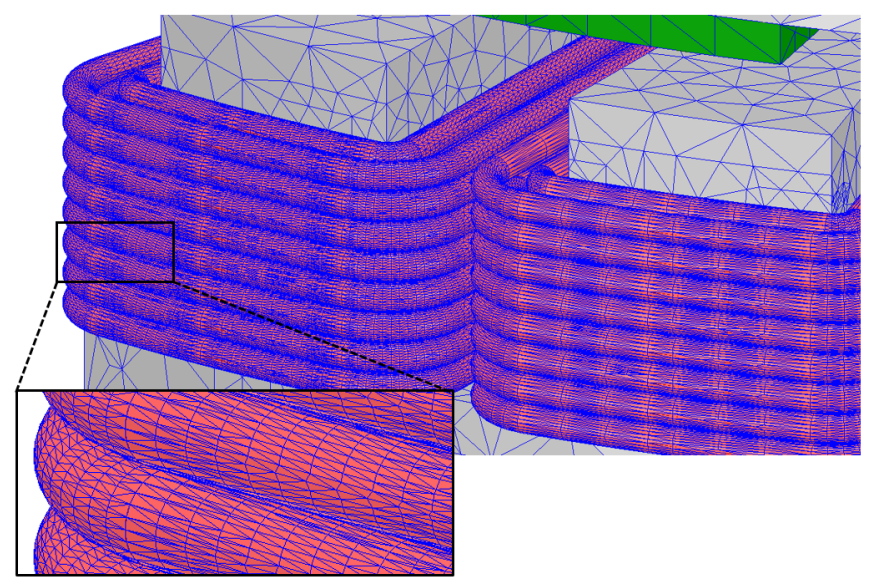

Fig. 9: The 3D mesh plot for the turn-by-turn model of the AFPM machine with open slots.

A coreless machine operating at higher frequencies would typically require the use of Litz wire, which is beyond the scope of the current paper.

The results for different methods presented in Table I include only the additional ac induced eddy current losses in the windings and not the steady-state dc-type loss component due to the main supply current. The four calculation methods considered are:

- 3D FEA: time-transient 3D FEA with detailed turn-byturn, i.e., wire-by-wire, representation of the winding coils (considered the most accurate method),

- Hy-3D: the new hybrid method with samples of flux density obtained from a simplified 3D model (the proposed method),

- 2D FEA: time-transient 2D FEA with turn-by-turn wireby-wire representation of the winding coils,

- Hy-2D: a hybrid method with samples from a simplified 2D FEA.

An example mesh plot used with the first method, the detailed time-stepping 3D FEA, is presented in Fig. 9. The hybrid methods studied, i.e., Hy-3D and Hy-2D, employed 
time-transient analysis. Nevertheless, in principle magnetostatic analysis and adaptive meshing may also be used, which could further reduce the computational time.

The additional eddy current losses calculated for the coreless machine example are significantly larger due to the winding conductors being exposed to the air-gap flux density. The results show increase in the additional winding loss for load operating conditions. This is because the varying flux density is not only originated from magnets, but also the ac current flowing in the neighboring conductors as well as the ac current inside the same conductor can induce additional eddy currents. Another reason may be attributed to the increased variation of the flux density due to armature reaction, causing distortion and asymmetry in flux lines.

The new Hy-3D method takes into account the effect of end turns in the return path of eddy currents through the use of the correction factor, $K_{s}$, introduced in (1). The calculated $K_{s}$ value is 0.98 for both machines, meaning that in these cases, the correction due to the end winding is minimal and the differences in results between methods may be substantially associated with the $3 \mathrm{D}$ field variation along the active sides of the coils.

In Table I, the first method listed, the 3D FEA, is considered as the most accurate approach and its results serve for reference. For the example open slot machine, the estimation differences for the Hy-3D, 2D FEA, and Hy-2D methods are $3 \%, 16 \%$, and $11 \%$ at open-circuit, respectively and $8 \%, 28 \%$, and $11 \%$ on load, respectively, illustrating the advantages of the new Hy-3D method in terms of satisfactory estimation. Although the new Hy-3D method takes longer to solve than 2D based algorithms, it is faster by one order of magnitude than the reference full 3D FEA and it is applicable for a wide range of problems. It should be noted that for the coreless machine studied, the 2D based methods simply fail to provide any estimations suitable even for basic engineering calculations.

\section{CONCLUSION}

This paper proposes a new hybrid analytical-FEA method for calculating ac eddy current losses in electrical machine windings with special application for axial flux designs, such as those of the PM synchronous type. The new method brings a major improvement above more conventional FEA based hybrid methods by considering the variation of the flux density in a 3D dimension, namely radial in the case of an axial flux machine and axial for a radial flux machine.

The computational results for two case studies of AFPM machines show that the new method is superior to other hybrid FE techniques in terms of accuracy and that its results satisfactorily compare with reference detailed 3D FEA, while reducing the computational time by one order of magnitude. The new method achieves a trade-off between speed and precision, making it suitable for different stages of the design process of an electrical machine.

\section{ACKNOWLEDGMENT}

The support of National Science Foundation NSF Grant \#1809876, of University of Kentucky, the L. Stanley Pigman endowment, and of ANSYS Inc. is gratefully acknowledged.

\section{REFERENCES}

[1] M. Popescu and D. G. Dorrell, "Proximity losses in the windings of high speed brushless permanent magnet ac motors with single tooth windings and parallel paths," IEEE Transactions on Magnetics, vol. 49, no. 7, pp. 3913-3916, July 2013.

[2] A. Fatemi, D. M. Ionel, N. A. O. Demerdash, D. A. Staton, R. Wrobel, and Y. C. Chong, "A computationally efficient method for calculation of strand eddy current losses in electric machines," in 2016 IEEE Energy Conversion Congress and Exposition (ECCE), Sep. 2016, pp. 1-8.

[3] M. Rosu, P. Zhou, D. Lin, D. M. Ionel, M. Popescu, F. Blaabjerg, V. Rallabandi, and D. Staton, IEEE Press Series on Power Engineering. IEEE, 2018. [Online]. Available: https://ieeexplore-ieeeorg.ezproxy.uky.edu/document/8233737

[4] C. R. Sullivan, "Computationally efficient winding loss calculation with multiple windings, arbitrary waveforms, and two-dimensional or threedimensional field geometry," IEEE Transactions on Power Electronics, vol. 16, no. 1, pp. 142-150, Jan 2001.

[5] P. B. Reddy, T. M. Jahns, and T. P. Bohn, "Modeling and analysis of proximity losses in high-speed surface permanent magnet machines with concentrated windings," in 2010 IEEE Energy Conversion Congress and Exposition, Sep. 2010, pp. 996-1003.

[6] L. J. Wu and Z. Q. Zhu, "Analytical investigation of open-circuit eddy current loss in windings of pm machines," in 2012 XXth International Conference on Electrical Machines, Sep. 2012, pp. 2759-2765.

[7] P. Mellor, R. Wrobel, D. Salt, and A. Griffo, "Experimental and analytical determination of proximity losses in a high-speed pm machine," in 2013 IEEE Energy Conversion Congress and Exposition, Sep. 2013, pp. 3504-3511.

[8] G. Volpe, M. Popescu, F. Marignetti, and J. Goss, "Modelling ac winding losses in a pmsm with high frequency and torque density," in 2018 ECCE, Sept 2018, pp. 2300-2305.

[9] O. A. Mohammed and S. Ganu, "FE-circuit coupled model of electric machines for simulation and evaluation of EMI issues in motor drives," IEEE Transactions on Magnetics, vol. 46, no. 8, pp. 3389-3392, Aug 2010.

[10] R. L. Russell and K. H. Norsworthy, "Eddy currents and wall losses in screened-rotor induction motors," Proceedings of the IEE - Part A: Power Engineering, vol. 105, no. 20, pp. 163-175, April 1958.

[11] J. R. Hendershot and T. J. E. Miller, Design of brushless permanentmagnet motors / J.R. Hendershot, Jr., T.J.E. Miller. Magna Physics Pub. ; Clarendon Press Hillsboro, OH : Oxford, 1994.

[12] R.-J. Wang and M. J. Kamper, "Calculation of eddy current loss in axial field permanent-magnet machine with coreless stator," IEEE Transactions on Energy Conversion, vol. 19, no. 3, pp. 532-538, Sep. 2004. 